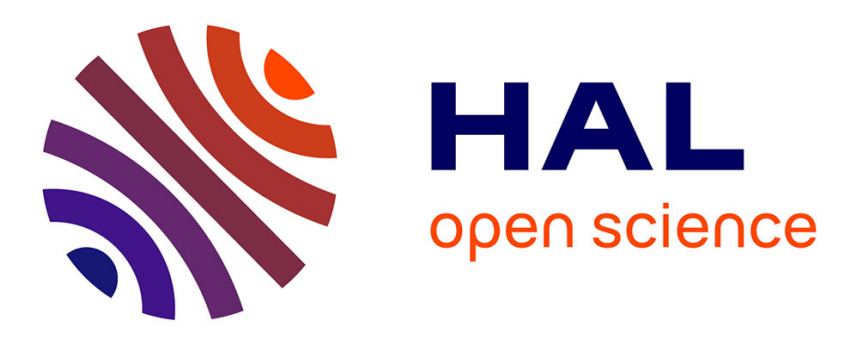

\title{
Analysis of limitations in divided-pulse nonlinear compression and amplification
}

Florent Guichard, Marc Hanna, Yoann Zaouter, Dimitris N. Papadopoulos, Frédéric Druon, Patrick Georges

\section{- To cite this version:}

Florent Guichard, Marc Hanna, Yoann Zaouter, Dimitris N. Papadopoulos, Frédéric Druon, et al.. Analysis of limitations in divided-pulse nonlinear compression and amplification. IEEE Journal of Selected Topics in Quantum Electronics, 2014, 20 (5), pp.7600405. 10.1109/JSTQE.2014.2313920 . hal-01069011

\section{HAL Id: hal-01069011 \\ https://hal-iogs.archives-ouvertes.fr/hal-01069011}

Submitted on 21 Apr 2016

HAL is a multi-disciplinary open access archive for the deposit and dissemination of scientific research documents, whether they are published or not. The documents may come from teaching and research institutions in France or abroad, or from public or private research centers.
L'archive ouverte pluridisciplinaire HAL, est destinée au dépôt et à la diffusion de documents scientifiques de niveau recherche, publiés ou non, émanant des établissements d'enseignement et de recherche français ou étrangers, des laboratoires publics ou privés. 


\title{
Analysis of Limitations in Divided-Pulse Nonlinear Compression and Amplification
}

\author{
Florent Guichard, Marc Hanna, Yoann Zaouter, Dimitrios N. Papadopoulos, Frédéric Druon, and Patrick Georges
}

\begin{abstract}
We analyze quantitatively physical effects that limit the coherent combining efficiency in highly nonlinear divided-pulse fiber-based setups such as parabolic amplifiers or nonlinear temporal compression experiment. The impact of differential dispersion and non-equal replicas power is assessed using numerical simulations and compared with experimental demonstrations. We also point out optimization strategies to minimize the impact of these effects on the performance of ultrafast fiber sources.
\end{abstract}

Index Terms-Coherent combining, fiber lasers, fiber nonlinear optics, ultrafast lasers.

\section{INTRODUCTION}

$\mathbf{U}$ LTRAFAST fiber laser sources have attracted much attention in the past decade due to their exceptional properties in terms of average power, beam quality, and robustness. The fiber geometry provides average power handling capability, but restricts the amplified pulse energy because of the small core area and large interaction length within the gain medium resulting in increased nonlinearities. Ytterbium-doped fiber amplifiers are of particular importance as high-power sources due to the very simple spectroscopic properties of the ytterbium ion. However, the gain bandwidth of ytterbium-doped glass only extends over $40 \mathrm{~nm}$ around the central wavelength of $1040 \mathrm{~nm}$, thereby limiting the amplified pulsewidth available. This effect, together with imperfect compression due to optical nonlinear effects, typically restricts the pulsewidths obtained from fiberamplified sources to the 300-500 fs range. These quantities, pulse energy and pulsewidth, constitute the remaining performance bottlenecks in high power ultrafast fiber sources.

Recently, coherent combining of several ultrafast sources has been implemented in the context of fiber amplifiers to overcome

Manuscript received November 29, 2013; revised January 28, 2014 and March 1, 2014; accepted March 24, 2014. This work was supported by the Agence Nationale de la Recherche through the Multifemto project.

F. Guichard is with the Laboratoire Charles Fabry de l'Institut d'Optique, UMR 8501 CNRS, Université Paris Sud, 91127 Palaiseau, France, and also with the Amplitude Systèmes, 33600 Pessac, France (e-mail: florent.guichard@ institutoptique.fr).

M. Hanna, F. Druon, and P. Georges are with the Laboratoire Charles Fabry de l'Institut d'Optique, UMR 8501 CNRS, Université Paris Sud, 91127 Palaiseau, France (e-mail: marc.hanna@institutoptique.fr; frederic. druon@institutoptique.fr; patrick.georges@institutoptique.fr).

Y. Zaouter is with Amplitude Systèmes, 33600 Pessac, France (e-mail: yzaouter@amplitude-systemes.com).

D. N. Papadopoulos is with the Laboratoire d'Utilisation des Lasers Intenses, Ecole Polytechnique, CNRS, CEA, UPMC, 91128 Palaiseau, France (e-mail: dimitrios.papadopoulos@polytechnique.edu).

Color versions of one or more of the figures in this paper are available online at http://ieeexplore.ieee.org.

Digital Object Identifier 10.1109/JSTQE.2014.2313920 these bottlenecks. Several architectures can be used. The first idea is to seed several fiber amplifiers with a common oscillator, and control actively the optical phase in each amplifier to combine the amplified beams while preserving the temporal and spatial properties of a single amplified beam [1], [2]. This allows, in principle, arbitrary scaling of the average power and pulse energy. Another possibility is to use setups where several amplified pulses are coherently added in a passive architecture, the phase being automatically matched because the optical path lengths experienced by different pulses are inherently equal. This can be done using two beams that counterpropagate in a Sagnac loop [3], [4]. It can also be achieved in the time domain using the divided-pulse (DP) principle [5]. Temporal and spatial passive approaches can also be combined to scale the number of replicas [6].

Although combining ideas were first applied to amplifier systems, it was very recently recognized that any energy-limited setup could benefit from these architectures. In particular, the DP concept was applied to nonlinear temporal post-compression setups using large-mode area glass fibers to induce self-phase modulation (SPM) [7], [8], and reach pulse durations below $100 \mathrm{fs}$. This allowed fiber-based systems delivering $70 \mathrm{fs}$ pulses with energies of $7 \mu \mathrm{J}$.

In this contribution, we provide an in-depth analysis of two phenomena that limit the coherent combining efficiency in highly nonlinear DP setups. Differential dispersion (DD), as explained hereafter, affects the combining efficiency of setups due to the fact that dividing and combining paths exhibit different dispersion properties. In the presence of nonlinearity, this breaks the symmetry of the division/combination processes, leading to a decrease in combining efficiency. Although identified in [8], this process is analyzed quantitatively here for the first time. The other main phenomenon that limits the efficiency is the nonequal intensities of the pulse replicas generated in the division process. SPM translates intensity changes into phase changes, hindering the passive combination process. For both effects, a quantitative study is made using numerical simulations, and the results are compared to the main experimental result obtained in the context of nonlinear post-compression [7]. The described effects are also encountered in stretcher-free amplifiers implementing the DP architecture [6]. Finally, we suggest possible approaches to minimize the nonlinear efficiency loss.

\section{EXPERIMENTAL SETUP}

We restrict the discussion to passive DP experiments that use an initially unchirped femtosecond pulse, divided into several replicas by propagation in a succession of highly birefringent 


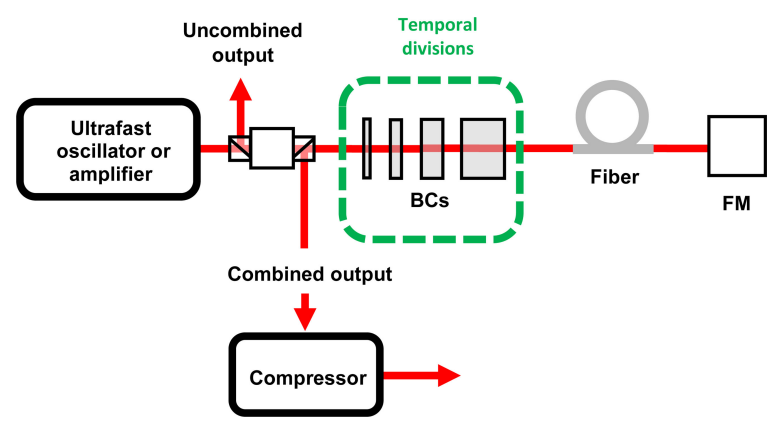

Fig. 1. Experimental setup for nonlinear parabolic amplifier or nonlinear compression experiments, BCs: birefringent crystals, FM: Faraday Mirror, Fiber: passive or diode-pumped active fiber.

crystals. This scheme can be applied to nonlinear parabolic amplifiers [6] or nonlinear compression setups [7], [8].

The experimental setups used in [6]-[8] are mostly equivalent, as depicted in Fig. 1. Starting with an ultrafast oscillator or amplifier, the pulses are divided in several temporal replicas by sending them in a sequence of birefringent crystals. In each crystal, the incident polarization state is linear and oriented at $45^{\circ}$ of the optical axes, generating at the output two temporally separated replicas of ideally equal amplitude and orthogonal polarization if the group delay difference between both axes is larger than the pulsewidth. The process can be repeated with $\mathrm{N}$ crystals, allowing the generation of $2^{N}$ temporal pulse replicas. Furthermore in this configuration, successive crystals should exhibit a length at least doubled from the previous one in order to avoid any temporal overlap between replicas.

After pulse division, the train of replicas is launched into a fiber. This fiber is either passive, to nonlinearly broaden the incident spectrum via SPM, or active, where the cumulated effects of gain, dispersion and nonlinearities contribute to broaden the incident spectrum. Recombination is then provided through a $90^{\circ}$ polarization rotation (e.g. using a Faraday rotator) which ensures a complementary propagation path inside the birefringent crystals on the way back, and thus a complete and self-balanced compensation of the previously induced delay between replicas. Although a Sagnac loop can also be used in these setups, its sole effect is to multiply the equivalent number of replicas by a factor of 2 , and it will not be considered in our analysis hereafter.

Possible phase mismatch between replicas can alter the coherent combining process. In the case of DP experiments, this corresponds to a fraction of power being directed towards the uncombined output of Fig. 1, and to the onset of parasitic pulses besides the main combined pulse in the time domain, [9]. The spatial efficiency is simply given by

$$
\eta_{\text {spatial }}=\frac{P_{\text {combined }}}{P_{\text {combined }}+P_{\text {uncombined }}}
$$

while the temporal combining efficiency is defined as

$$
\eta_{\text {temporal }}=\frac{E_{\text {peak }}}{E_{\text {peak }}+\sum E_{\text {parasitic }}}
$$

where $E_{\text {peak }}$ and $E_{\text {parasitic }}$ correspond respectively to the energies of the main recombined pulse and of parasitic replicas on

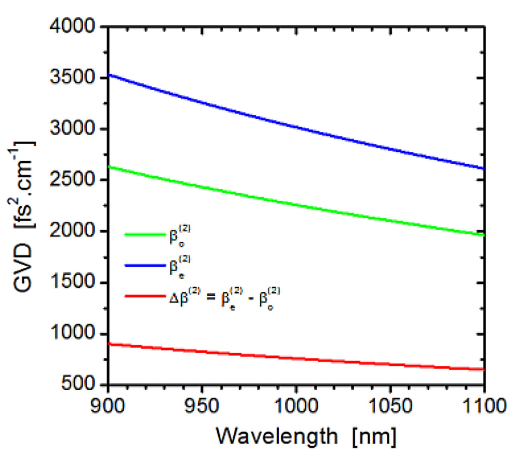

Fig. 2. Group-velocity dispersion along the extraordinary (blue) and ordinary (green) axes in $\mathrm{YVO}_{4}$ crystal and differential GVD (red).

the combined output. Experimentally, this efficiency is easily measured from the output autocorrelation trace. The autocorrelation value at zero delay is divided by the sum of autocorrelation peaks on a single temporal side, including the zero delay value. The total combining efficiency is finally given by the product:

$$
\eta=\eta_{\text {spatial }} \times \eta_{\text {temporal }}
$$

\section{DIFFERENTIAL DISPERSION}

Although the role of the birefringent crystals is to introduce a large group delay between both polarization components, the higher order spectral phase induced by propagation through the crystals are also different on both axes. This effect is denoted here as DD. Fig. 2 shows group-velocity dispersions (GVD), calculated from [10], accumulated during propagation on the extraordinary ( $e$-axis) and ordinary ( $o$-axis) axes in an yttrium vanadate crystal $\left(\mathrm{YVO}_{4}\right)$ used in the above-mentioned experiments. The second-order DD has a typical value of $725 \mathrm{fs}^{2} . \mathrm{cm}^{-1}$ at $1030 \mathrm{~nm}$. In the case of a strictly linear setup, i.e. if the incident spectral shape is unchanged during the experiment, this DD has no impact on the combining efficiency because all the pulses experience the exact same optical path upon division and recombination. Indeed, the DD accumulated between two replicas on the way forward is experienced with an opposite sign on the way backward.

However, in the case of a nonlinear setup, where large spectral broadening occurs between the division and combination steps, the DD of spectral orders greater than 2 (group-velocity dispersion and higher orders) experienced by newly created spectral content is not compensated. This induces a spectral phase that decreases the coherent combining efficiency, depending both on crystal properties and accumulated nonlinearities.

To gain more insight on this effect and evaluate its quantitative impact on the coherent combining efficiency, we perform numerical simulations. Propagation is supposed to be linear in yttrium vanadate crystals, and all orders of dispersion are taken into account by using a Sellmeier model for the wavelengthdependent refractive index. A Jones matrix formalism is used to take into account polarization rotation between crystals. The propagation in the fiber is modeled with a vector split-step Fourier algorithm [11] taking into account dispersion up to third order, SPM, and cross-phase modulation between the polariza- 

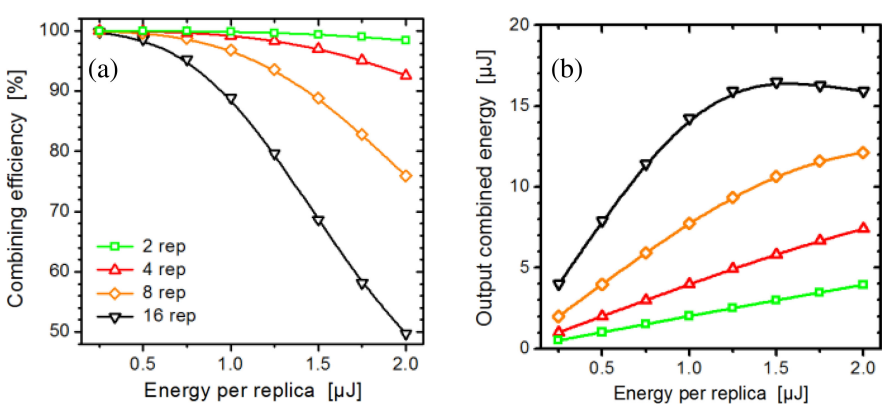

Fig. 3. (a) Combining efficiency as a function of energy per replica for respectively 2 (green), 4 (red), 8 (orange), and 16 (black) numbers of replicas. (b) Corresponding total output energy for 2, 4, 8 and 16 replicas.
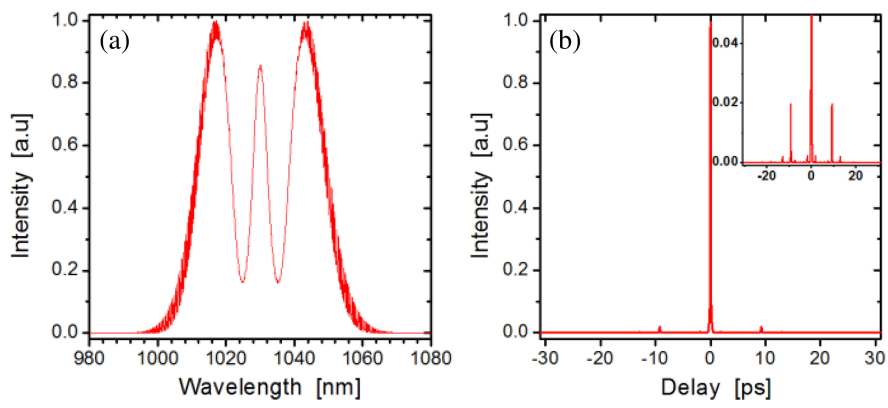

Fig. 4. (a) Combined output spectrum for 16 temporal replicas at $1 \mu \mathrm{J} /$ replica, corresponding to a combining efficiency of $\eta=89 \%$. (b) Corresponding autocorrelation trace. Insert: Emphasis on the amplitude interval [0 0.05].

tion states. The initial condition is a single $300 \mathrm{fs}$ Gaussian pulse, and is divided in a maximum of 16 temporal replicas through the propagation in a series of four a-cut $\mathrm{YVO}_{4}$ crystals with lengths 2.5, 5, 10 and $20 \mathrm{~mm}$. Nonlinear effects then occur in a fiber with a mode field diameter of $45 \mu \mathrm{m}$. In order to only evaluate the DD effect, we suppose here the generation of replicas with exactly equal powers.

Results are displayed in Fig. 3, showing the evolution of the combining efficiency and total output energy, for different number of replicas, with increasing nonlinearities, i.e. energy per replica. This energy per replica is limited to $2 \mu \mathrm{J}$ because detrimental self-focusing and damage at the fiber facet typically appear above $1 \mu \mathrm{J}$ for the experiment reported in ref. [7]. It clearly shows that the efficiency drops when the input energy increases owing to the increasing spectral content generated by SPM. Moreover, the efficiency decay is more important when the number of replicas is increased, resulting in efficiencies of $\eta=99.8 \%$ and $\eta=89 \%$ at $1 \mu \mathrm{J}$ per replica for 2 and 16 temporal replicas respectively. This is easily understood because the propagation length inside the crystals, and consecutively the DD, is increased when the number of generated replicas increases. The decrease in efficiency is also visible on the output recombined spectrum (see Fig. 4) with the onset of spectral interferences on both spectrum sides. Indeed, as previously mentioned, the common spectral part of the input and output spectrum is not sensitive to DD since the double pass in the crystals cancels it.

DD thus appears as an intrinsic limiting factor in such experiments, fixing an upper value of the combining efficiency, depending on the crystals properties and nonlinearity level. Nev-

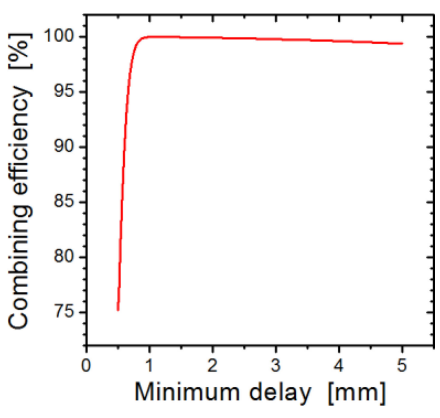

Fig. 5. Combining efficiency as a function of crystal length, i.e. minimum induced-delay, for 2 replicas and $1 \mu \mathrm{J} /$ replica.

ertheless, it is possible to mitigate this effect by minimizing the propagation length inside the crystals. First, the lengths of successive crystals should be exactly doubled to minimize the overall length of material. Second, the length of the smallest crystal should be as small as possible while avoiding detrimental effects caused by the overlap of successive pulses. We have investigated this minimum length in simulations by computing the combining efficiency as a function of crystal length in a setup with only 2 replicas, as shown in Fig. 5. Two trends clearly appear. For crystal lengths above $1 \mathrm{~mm}$ the combining efficiency linearly decreases because of increasing DD. For lengths below $1 \mathrm{~mm}$, a large and quick decay of the efficiency appears corresponding to a temporal overlap between replicas leading to cross-phase modulation-induced (or SPM-induced in the case of a system incorporating a Sagnac loop) spectral phases that decrease the combining efficiency. The tradeoff length, for an input pulsewidth of $300 \mathrm{fs}$ and $1 \mu \mathrm{J} / \mathrm{replica}$ is $1.09 \mathrm{~mm}$, which would lead to combining efficiencies above $99 \%$ in a 16 replicas setup.

Although this study is focused on DP experiments using birefringent crystals, it is also relevant in the case of DP experiments using polarizers and delay-lines as replicas generator. Indeed, DD may also affect the combining efficiency since propagation in a thin-film polarizer (TFP) induces a DD between the $p$-polarized replicas (transmitted by the TFP) and the $s$-polarized replicas (reflected by the TFP). The order of magnitude of this differential GVD is $200 \mathrm{fs}^{2} \cdot \mathrm{cm}^{-1}$ at $1030 \mathrm{~nm}$ between air and fused-silica. Although this leads to an overall DD that is much lower than crystals, it could still limit the efficiency of DP setups using very short pulses, for example in a capillary basednonlinear compression setup for sub-10 fs pulse generation from Ti:Sa laser sources. However, in this case, the optical beams are separated in space, giving access to the individual optical paths followed by each replicas. It is therefore possible to balance the dispersion, or any other non-symmetric property (e. g. intensity) by inserting optical elements in the delay lines. For instance, a fused silica plate might be inserted in the delay line to exactly balance the dispersion introduced by TFPs. The main drawback of freespace delay lines is the difficulty of optical alignment and lack of compactness when the number of pulse replicas is increased. 

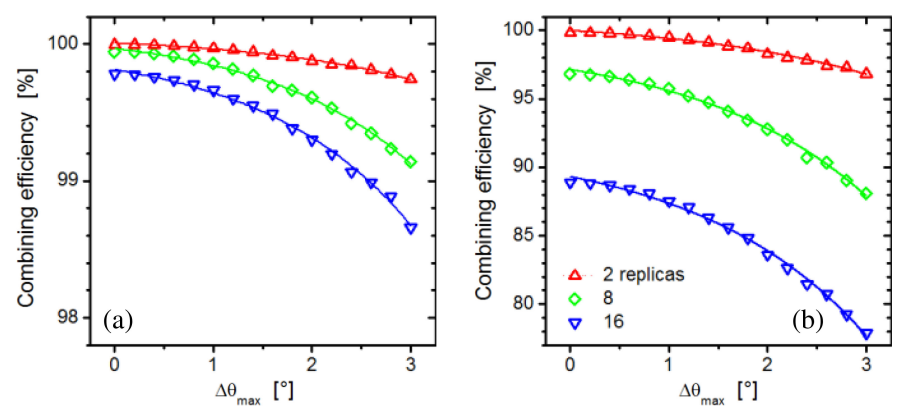

Fig. 6. Combining efficiency as a function of angular precision $\Delta \theta_{\max }$ for $0.25 \mu \mathrm{J}$ (a) and $1 \mu \mathrm{J}$ (b) per replica, for respectively 2 (red), 8 (green) and 16 (blue) replicas.

\section{REPLICA POWERS EQUALIZATION}

We have identified another effect that contributes to decrease the combining efficiency in practical DP nonlinear experiments. Indeed, reported experimental efficiencies are well below the DD-induced efficiency calculated in previous section. As an example, the DD-induced efficiency is $89 \%$ for 16 replicas at $1 \mu \mathrm{J} /$ replicas with crystal lengths corresponding to the experiment reported in ref. [7]. However the experimentally obtained efficiency is around $70 \%$. In this experiment, due to the implementation of a Sagnac interferometer, an additional decrease in the combining efficiency is related to imperfect spatial matching of both counter-propagating beams. This decrease in combining efficiency is evaluated to $8 \%$, which is not sufficient to account for the total combining efficiency decrease.

In the specific case of nonlinear DP stretcher-free amplification experiments, where the energy per replica is well below the gain saturation level, the replicas amplitudes and phases are not affected by differential gain and the related Kramers-Krönig phase shift [12]. Furthermore, in nonlinear temporal compression experiments, these effects are not relevant due to the absence of gain. However, replicas can be generated with slightly different amplitudes because of experimental imperfections of the dividing setup. In strongly nonlinear experiments, the slight amplitude variation is translated through SPM into phase variations that prevent perfect coherent combining. In a crystal-based division setup, the crystal orientation is adjusted with a finite precision that translates directly into amplitude variation. To quantify this effect, we have performed simulations in which the orientations of the successive dividing/combining crystals differ from the ideal case by a random amount $\Delta \theta$. For each crystal, this angle is taken as a random variable with uniform distribution in the interval [ $\left.-\Delta \theta_{\max } / 2 \Delta \theta_{\max } / 2\right]$. The combining efficiency is computed for 200 realizations of these angles, and the average value of efficiency is computed following the Monte-Carlo method. This simulation also includes the effect of DD discussed in previous section.

Fig. 6 shows the evolution of average combining efficiency as a function of $\Delta \theta_{\max }$ for an increasing number of replicas at two energy levels per replica, respectively $0.25 \mu \mathrm{J}$ [Fig. 6(a)] and $1 \mu \mathrm{J}$ [Fig. 6(b)]. There is a clear efficiency drop as the uncertainty on the crystal orientation is increased, with an obtained value of $78 \%$ for $1 \mu \mathrm{J} /$ replica, a total number of 16 replicas, and an
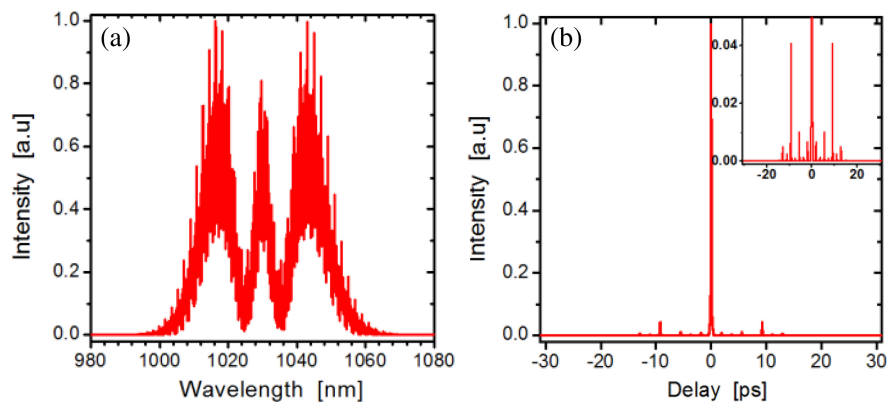

Fig. 7. (a) Typical combined output spectrum for $\Delta \theta_{\max }=3^{\circ}$, and 16 temporal replicas at $1 \mu \mathrm{J} /$ replica, corresponding to a combining efficiency of $\eta=$ $77 \%$. (b) Corresponding autocorrelation trace. Insert: Emphasis on the amplitude interval $\left[\begin{array}{ll}0 & 0.05\end{array}\right]$.

angle precision of $\Delta \theta_{\max }=3^{\circ}$. Note that this angular precision corresponds to a power variation of approximately only $\pm 5 \%$. The efficiency also decreases with the number of generated replicas. The impact of the non-equal powers of replicas is also clearly visible on the recombined output compressed pulse, as shown in Fig. 7. The output autocorrelation and spectrum are plotted for a total combining efficiency of $77 \%, 16$ replicas, and $1 \mu \mathrm{J} /$ replica. Here, spectral interferences (and thus parasitic replicas) appear over the whole spectrum, because the nonlinear phase shift is susceptible to vary between two replicas and thus the spectral phase varies over the entire spectrum.

Taking into account the Sagnac interferometer spatial efficiency of $92 \%$, the angle uncertainty that corresponds to the experimental value of $70 \%$ in [7] is $\Delta \theta_{\max }=3^{\circ}$ with 16 replicas at $1 \mu \mathrm{J} /$ replica, corresponding to a standard deviation of

$$
\sigma_{\theta}=\frac{1}{2 \sqrt{3}} \cdot \Delta \theta_{\max }=0.87^{\circ} .
$$

Equalization of the replicas power is therefore an important source of efficiency decrease in highly nonlinear DP setups.

\section{CONCLUSION}

The DP architecture has proven a valuable tool to scale the energy of fiber-based femtosecond sources and nonlinear compression setups. We have identified two physical effects that limit the combining efficiency of this concept in the presence of optical nonlinearities. For given target performances of a source in terms of pulse duration and energy, a compromise must be found between the level of nonlinearity, given by the energy per replica, and the number of replicas generated by the system. In a nonlinear compression setup, the energy per replica determines the achievable output pulse duration, but cannot be increased arbitrarily because it reduces the tolerance to non-equal replica powers. The number of replicas that can be used is limited by the DD effect, which also depends on the nonlinearity level. Possible solution to the above is the implementation of free-space delay lines that can provide dispersion-balanced operation. The cost in this case is the increased complexity of the setup especially for large number of replicas. The optimal system is the result of these physical and practical limitations, and is largely dependent on the specifics of the setup. Of particular importance 
is the potential implementation of DP compression using gasfilled capillary-based compression setups for few-cycle pulse generation.

\section{REFERENCES}

[1] L. Daniault, M. Hanna, L. Lombard, Y. Zaouter, E. Mottay, D. Goular, P. Bourdon, F. Druon, and P. Georges, "Coherent beam combining of two femtosecond fiber chirped-pulse amplifiers," Opt. Lett., vol. 36, pp. 621623, 2011.

[2] E. Seise, A. Klenke, J. Limpert, and A. Tünnermann, "Coherent addition of fiber-amplified ultrashort laser pulses," Opt. Exp., vol. 18, pp. $27827-$ 27835, 2010.

[3] S. Roither, A. J. Verhoef, O. D. Mücke, G. A. Reider, A. Pugzlys, and A. Baltuska, "Sagnac interferometric multipass loop amplifier," Opt. Exp., vol. 20, pp. 25121-25129, 2012.

[4] Y. Zaouter, L. Daniault, M. Hanna, D. N. Papadopoulos, F. Morin, C. Hönninger, F. Druon, E. Mottay, and P. Georges, "Passive coherent combination of ultrafast rod type chirped pulse amplifiers," Opt. Lett., vol. 37, pp. 1460-1462, 2012.

[5] S. Zhou, D. G. Ouzounov, and F. W. Wise, "Divided-pulse amplification of ultrashort pulses," Opt. Lett., vol. 32, pp. 871-873, 2007.

[6] L. Daniault, M. Hanna, D. N. Papadopoulos, Y. Zaouter, E. Mottay, F. Druon, and P. Georges, "High peak-power stretcher-free femtosecond amplifier using passive spatio-temporal coherent combining," Opt. Exp., vol. 20, pp. 21627-21634, 2012.

[7] F. Guichard, Y. Zaouter, M. Hanna, F. Morin, C. Hönningerning, E. Mottay, F. Druon, and P. Georges, "Energy scaling of a nonlinear compression setup using passive coherent combining," Opt. Lett., vol. 38, pp. $4437-4440,2013$.

[8] A. Klenke, M. Kienel, T. Eidam, S. Häddrich, J. Limpert, and A. Tünnermann, "Divided-pulse nonlinear compression," Opt. Lett., vol. 38, pp. 4593-4596, 2013.

[9] L. J. Kong, L. M. Zhao, S. Lefrançois, D. G. Ouzounov, C. X. Yang, and F. W. Wise, "Generation of megawatt peak power picosecond pulses from a divided-pulse fiber amplifier," Opt. Lett., vol. 37, pp. 253-255, 2012.

[10] M. Bass, C. DeCusatis, J. Enoch, V. Lakshminarayanan, G. Li, C. MacDonald, V. Mahajan, and E. V. Stryland, Handbook of Optics. New York, NY, USA: McGraw Hill, 2009.

[11] G. P. Agrawal, Nonlinear Fiber Optics, 4th ed. New York, NY, USA: Academic, 2007.

[12] M. Kienel, A. Klenke, T. Eidam, M. Baugmartl, C. Jauregui, J. Limpert, and A. Tünnermann, "Analysis of passively combined divided-pulse amplification as an energy-scaling concept," Opt. Exp., vol. 21, pp. 2903129042, 2013.

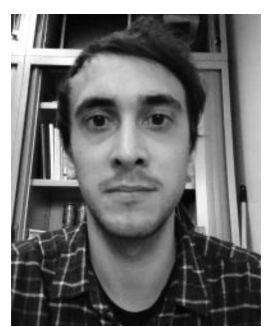

Florent Guichard received the Diploma of Laser, Matter and Nanoscience, Master's degree in physics, from the University of Bordeaux 1, Talence, France, in 2011. Since 2012, he has been working toward the Ph.D. degree, in collaboration with the company Amplitude Systèmes, Pessac - France, at the Laboratoire Charles Fabry de l'Institut d'Optique, Centre National de la Recherche Scientifique (CNRS), Université Paris Sud, Palaiseau, France. His current research interests are focused on ultrafast and highpower fiber lasers and coherent combining concepts to scale the average power and energy of ultrafast fiber systems.

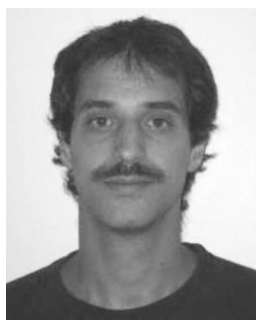

Marc Hanna received the engineering degree from the Institut National des Télécommunications, Evry, France, in 1996, and the Ph.D. degree in electrical engineering from the Université de Franche Comté, Besançon, France, in 2000. From 2001 to 2006, he was a Research Scientist in the Optics Department, Franche-Comté Electronique, Mécanique, Thermique et Optique-Sciences et Technologies (FEMTO-ST), Besançon, where he focused on ultrafast optics applied to telecommunications. He is currently with the Laboratoire Charles Fabry de l'Institut d'Optique, Centre National de la Recherche Scientifique (CNRS), Université Paris Sud, Palaiseau, France. His research interests include ultrafast and nonlinear optics, fiber lasers and amplifiers, and coherent addition of ultrafast optical fields.

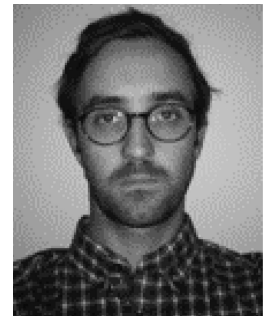

Yoann Zaouter received an engineering degree in optronics from IFIPS - Université Paris Sud, Orsay, France in 2004, a Master of Science degree in optics and photonics from Université Paris Sud, Orsay, France, in 2005 and a Ph.D. degree in Laser, Matter and Nanoscience from Université de Bordeaux, Bordeaux, France in 2008. His Ph.D work was carried out in collaboration with the company Amplitude Systemes, Pessac, France, and was focused on the development of novel ultrafast fiber laser architectures for industrial and scientific applications. In 2008, he joins the research and development department of Amplitude Systemes and developed the first generation of industrial ultrafast fiber lasers. Since 2009, he heads a joint research and development laboratory shared with the laser development team at Laboratoire Charly Fabry, Institut d'Optique, Palaiseau, France, where he develops laser concepts for average power and energy scaling of ultrafast laser technologies. His current research interests include ultrafas and nonlinear optics, fiber lasers and amplifiers, active and passive coherent combining of ultrafast amplifiers.

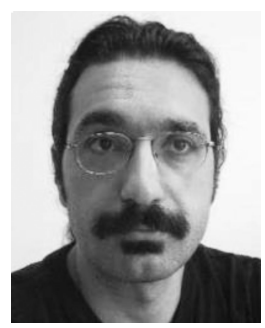

Dimitrios N. Papadopoulos received the diploma in electrical and computer engineering in 1998, the Master's degree in physics, and the Ph.D. degree in laser physics from the National Technical University of Athens (NTUA), Athens, in 2005. From 2007 to 2009, he was a Postdoctoral Researcher at the Laboratoire Charles Fabry de l'Institut d'Optique, Palaiseau, France on ultrafast laser oscillators and high-power femtosecond fiber amplifiers. Since 2009, he has been a Research Engineer for the Apollon 10P project (LULI/Ecole Polytechnique, Palaiseau, France). His current research interests include the generation of few cycle pulses, OPCPA techniques, high energy diode pumped systems and Ti:Sapphire amplifiers.

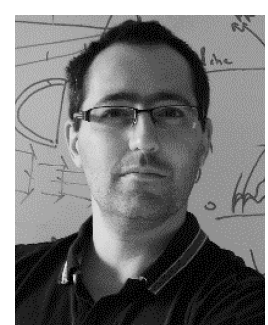

Frédéric Druon received the engineer degree from the Electrical Engineering School of Paris (Supélec), Gif-sur-Yvette, France, in 1996, and the Ph.D. degree from the Institut d'Optique, Orsay, France, in 2000 , for work on ultracompact and efficient picosecond UV sources based on microchip lasers and on femtosecond diode-pumped laser systems. He studied optics and photonics at the University of Paris XI. In 1997, he made his military service as a Research Visitor at the Center for Ultrafast Optical Science (CUOS), Ann Arbor, MI, where he worked on wave-front analysis and correction of terawatt laser chains. He was a Postdoctoral Researcher for one year and worked on mode-locking processes involving fast saturable absorbers. In 2001, he joined the Laboratoire Charles Fabry de l'Institut d'Optique, Centre National de la Recherche Scientifique (CNRS) Universite Paris Sud, Palaiseau, France. His current research interests consist in innovative ultrafast laser development. He received the "Fabry - De Gramont" Prize in 2005 and the "Jean Jerphagnon" Prize in 2011 for his research.

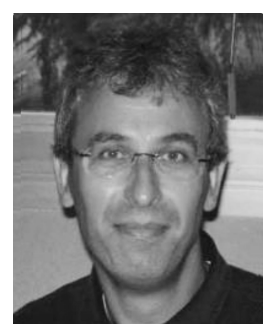

Patrick Georges received the engineer degree from the Ecole Supérieure d'Optique, Palaiseau, France, in 1985, and the Ph.D. degree from the Institut d'Optique, Orsay, France, in 1989, for work on colliding pulses mode-locked dye lasers at different wavelengths and pulse compression. He is currently a Senior Scientist at the Centre National de la Recherche Scientifique (CNRS), where he leads the Lasers Group, Laboratoire Charles Fabry at the Institut d'Optique, Palaiseau. His current research interests include diode-pumped solid-state lasers, new laser materials, picosecond and femtosecond lasers, high brightness laser diodes, fiber amplifier systems and optically pumped vertical surface emitting lasers. $\mathrm{He}$ is also engaged in the Apollon $10 \mathrm{PW}$ laser facility project and responsible of the Front-end. Dr. Georges is a Fellow Member of the Optical Society and has served as Program Chair for the Cleo Europe 2013 conference. 